\begin{tabular}{ccc}
\hline Bentham OPEN & The Open Statistics \& Probability \\
CrossMark & Content list available at: www.benthamopen.com/TOSPJ/ \\
\hline
\end{tabular}

RESEARCH ARTICLE

\title{
Some Characterization and Relations Based on K-th Lower Record Values
}

\author{
Ali A. Al-Shomrani* \\ Department of Statistics, Faculty of Science, King Abdulaziz University, Jeddah, Saudi Arabia
}

\begin{abstract}
In this paper, we obtain certain expressions and recurrence relations for two general classes of distributions based on some conditional expectations of $\mathrm{k}$-th lower record values. We consider the necessary and sufficient conditions such that these conditional expectations hold for some distribution functions. Furthermore, an expression of conditional expectation of other general class of distributions through truncated moments of some random variable is considered. Some distributions as examples of these general classes are shown in Tables $\mathbf{1}$ and $\mathbf{2}$ accordingly.
\end{abstract}

Keywords: Characterization, Conditional expectation, Order statistics, Records values.

\section{INTRODUCTION}

Record values are found in many real lifetime datasets such as observing new records in k-th highest or k-th lowest values of weather-conditions, temperatures, water-levels, Olympic or world records in sports. In addition, record values are used in theory of reliability. Furthermore, these statistics are related to the occurrence times of some nonhomogenous Poisson process used in shock models Kamps [1]. Chandler [2] started the statistical study of record values as a model considering dependence structure for successive extremes in a sequence of independent and identically distributed (iid) random variable. This means that, the life-length distribution of system components may change after the failure of each component. Dziubdziela and Kopocinski [3] proposed the limiting distribution of k-th record values where $\mathrm{k}$ is some positive integer. Many authors have considered characterization of distributions through conditional expectation of record values, for instance, Nagaraja [4], Franco and Ruiz [5, 6], Khan and Alzaid [7], Khan, Faizan, and Haque [8], and Lopez-Blazques and Moreno-Rebollo [9]. For more information in the theory of records and its distributional properties and some characterizations of $\mathrm{k}$-th record values can be found in, for example, Ahsanullah [10, 11], Arnold, Balakrishnan, and Nagaraja [12], Nevzorov [13], Deheuvels [14], Nagaraja [15], Raqab and Awad [16] and references therein.

Let $\left\{X_{i}, i \geq 1\right\}$ be a sequence of independently and identically distributed (iid) random variables with cumulative distribution function $(c d f) F(x)$ and probability density function $(p d f) f(x)$. For a fixed $k \geq 1$, the kth lower record value of $X_{i}^{\prime} s$ is defiend by:

$$
Z_{r}^{(k)}=X_{k: L_{r}^{(k)}+k-1} \text { where } L_{1}^{(k)}=1, L_{r+1}^{(k)}=\min \left\{j>L_{r}^{(k)}: X_{k: L_{r}^{(k)}+k-1}>X_{k: j+k-1}\right\} \text { for } r \geq 1
$$

Note that $Z_{1}^{(k)}=\max \left\{X_{1}, \cdots, X_{k}\right\}=X_{k: k}$ and $Z_{r}^{(1)}=X_{L_{r}}$ for $r \geq 1$ with

$$
L_{r}=\min \left\{j>L_{r-1}: X_{j}<X_{L_{r-1}}\right\} \text { are lower record values. }
$$

\footnotetext{
* Address correspondence to this author at the Department of Statistics, Faculty of Science, King Abdulaziz University, PO Box 80203, Jeddah 21589, Saudi Arabia; Tel: +966504379305; E-mail: email2aaalshomrani@yahoo.com
} 
For $\alpha<y<x<\beta, 1 \leq r<s \leq n, H(x)=-\log (F(x))$ and $h(x)=-H^{\prime}(x)=\frac{f(x)}{F(x)}$, we have the following (see Ahsanullah [10, 11], Arnold, Balakrishnan, and Nagaraja [12], Nevzorov [13]:

The pdf of $Z_{r}^{(k)}$ and $\left(Z_{r}^{(k)}, Z_{s}^{(k)}\right)$ are as follows:

$$
\begin{gathered}
f_{Z_{r}^{(k)}}(x)=\frac{k^{r}}{\Gamma(r)}(H(x))^{r-1}(F(x))^{k-1} f(x) \text { for } r \geq 1 \\
f_{Z_{r}^{(k)}, Z_{s}^{(k)}}(x, y)=\frac{k^{s}}{\Gamma(r) \Gamma(s-r)}(H(y)-H(x))^{s-r-1}(H(x))^{r-1}(F(y))^{k-1} h(x) f(y)
\end{gathered}
$$

We shall denote:

$$
\begin{gathered}
\mu_{m+1 \mid m}^{(k)}=E\left(g\left(Z_{m+1}^{(k)}\right) \mid Z_{m}^{(k)}=x\right)=\frac{k}{[F(x)]^{k}} \int_{\alpha}^{x} g(y)[F(y)]^{k-1} f(y) d y \\
\mu_{m \mid m+1}^{(k)}=E\left(g\left(Z_{m}^{(k)}\right) \mid Z_{m+1}^{(k)}=y\right)=\frac{m}{[H(y)]^{m}} \int_{y}^{\beta} g(x)[H(x)]^{m-1} h(x) d x \\
E[g(X) \mid X \leq x]=\frac{1}{F(x)} \int_{\alpha}^{x} g(w) f(w) d w
\end{gathered}
$$

Such that $g$ is a continuous, monotonic and differentiable function on $(\boldsymbol{\alpha}, \boldsymbol{\beta})$.

\section{MAIN RESULTS}

In this paper, we present three general classes of distributions whose cdf's are:

$$
\begin{gathered}
F(x)=\left[a e^{-b g(x)}\right]^{c}, \quad a, b \text { and } c \neq 0 \\
F(x)=\left[a+b e^{-c g(x)}\right]^{d}, \quad b, c \text { and } d \neq 0 \\
F(x)=a+b[c+d g(x)]^{p}, \quad b, d \text { and } p \neq 0
\end{gathered}
$$

such that $g$ is a continuous, monotonic and differentiable function on $(\boldsymbol{\alpha}, \boldsymbol{\beta})$.

We extend using the cdf in (6) some work of Al-Shomrani and Shawky [17] as shown in Theorem 1 by characterizing this first general form of distributions through conditional expectation of p-th power of difference of functions of two k-th lower record values. Moreover, Theorems 2.5 and 2.6 in Shawky and Abu-Zinadah [18] and Theorems 3 and 4 in Shawky and Bakoban [19] are generalized as shown in Theorems 2 and 3 using the cdf in (7) by characterizing the second general class of distributions through conditional expectation of k-th lower record values. Lastly, we show that equation (2.1.1) in Hamedani, Javanshiri, Maadooliat, and Yazdani [20] is a special case of Theorem 4 by using the cdf in (8) as the third general class of distributions based on truncated moments of some random variable. Some distributions as members of these general classes are given as examples in Tables $\mathbf{1}$ and $\mathbf{2}$.

\section{Theorem 1:}

Let $\mathrm{X}$ be an absolutely continuous random variable with $\operatorname{cdf} \mathrm{F}(\mathrm{x})$ and $\operatorname{pdf} \mathrm{f}(\mathrm{x})$ on the support $(\boldsymbol{\alpha}, \boldsymbol{\beta}), F(\alpha)=0$ and $F(\beta)=1$. Then, for two values of $r$ and $s, 1 \leq \mathrm{r}<\mathrm{s} \leq \mathrm{n}\left(\right.$ where $Z_{r}^{(k)}=X_{k: L_{r}^{(k)}+k-1}$ as defined above) 
Table 1. Examples of (7).

\begin{tabular}{|c|c|c|c|c|c|c|}
\hline Distribution & $F(x)$ & $g(x)$ & $\mathbf{a}$ & $\mathbf{b}$ & $\mathbf{c}$ & d \\
\hline Weibull & $1-e^{-\theta x^{p}}, \quad x>0$ & $x^{p}$ & 1 & -1 & $\theta$ & 1 \\
\hline Pareto of the first kind & $1-\lambda^{p} x^{-p}, x>\lambda$ & $\ln x$ & 1 & $-\lambda^{p}$ & $\mathrm{p}$ & 1 \\
\hline Burr XII & $1-\left(1+\theta x^{p}\right)^{-\lambda}, x>0$ & $\ln \left(1+\theta x^{p}\right)$ & 1 & -1 & $\lambda$ & 1 \\
\hline Rayleigh & $1-e^{-\theta x^{2}}, \quad x>0$ & $x^{2}$ & 1 & -1 & $\theta$ & 1 \\
\hline Lomax & $1-(1+\theta x)^{-\lambda}, x>0$ & $\ln (1+\theta x)$ & 1 & -1 & $\lambda$ & 1 \\
\hline Inverse Weibull & $e^{-\theta x^{-p}}, x>0$ & $x^{-p}$ & 0 & 1 & $\theta$ & 1 \\
\hline Power function & $\left(\frac{x}{\lambda}\right)^{p}, 0<x<\lambda$ & $\ln \left(\frac{x}{\lambda}\right)$ & 0 & 1 & $-p$ & 1 \\
\hline Rectangular & $\left(\frac{x-\beta}{\lambda-\beta}\right), \beta<x<\lambda$ & $\ln (x-\beta)$ & 0 & $\frac{1}{\lambda-\beta}$ & -1 & 1 \\
\hline Pareto of the second kind & $1-(1+x)^{-\lambda}, x>0$ & $\ln (1+x)$ & 1 & -1 & $\lambda$ & 1 \\
\hline Exponential & $1-e^{-\lambda x}, \quad x>0$ & $x$ & 1 & -1 & $\lambda$ & 1 \\
\hline Inverse Exponential & $e^{-\frac{\lambda}{x}}, x>0$ & $\frac{1}{x}$ & 0 & 1 & $\lambda$ & 1 \\
\hline Kumaraswamy & $1-\left(1-x^{p}\right)^{\lambda}, 0<x<1$ & $\ln \left(1-x^{p}\right)$ & 1 & -1 & $-\lambda$ & 1 \\
\hline Exponentiated Frechet & $1-\left(1-e^{-x^{-\alpha}}\right)^{\theta}, x>0$ & $\ln \left(1-e^{-x^{-\alpha}}\right)$ & 1 & -1 & $-\theta$ & 1 \\
\hline Exponentiated exponential & $\left(1-e^{-\lambda x}\right)^{\theta}, x>0$ & $\ln \left(1-e^{-2 x}\right)$ & 0 & 1 & $-\theta$ & 1 \\
\hline Burr X (Exponentiated Rayleigh) & $\left(1-e^{-\beta x^{2}}\right)^{\alpha}, x>0$ & $x^{2}$ & 1 & -1 & $\beta$ & $\alpha$ \\
\hline Exponentiated Weibull & $\left(1-e^{-\beta x^{p}}\right)^{\alpha}, x>0$ & $x^{p}$ & 1 & -1 & $\beta$ & $\alpha$ \\
\hline
\end{tabular}

Table 2. Examples of (8).

\begin{tabular}{|c|c|c|c|c|c|c|c|}
\hline Distribution & $F(x)$ & $g(x)$ & a & b & c & d & p \\
\hline Weibull & $1-e^{-\theta x^{\beta}}, x>0$ & $e^{-\theta x^{\beta}}$ & 0 & 1 & 1 & -1 & 1 \\
\hline Pareto of the first kind & $1-\lambda^{\beta} x^{-\beta}, x>\lambda$ & $\frac{1}{x}$ & 1 & -1 & 0 & $\lambda$ & $\beta$ \\
\hline Burr XII & $1-\left(1+\theta x^{p}\right)^{-\lambda}, x>0$ & $x^{p}$ & 1 & -1 & 1 & $\theta$ & $-\lambda$ \\
\hline Rayleigh & $1-e^{-\theta x^{2}}, \quad x>0$ & $e^{-\theta x^{2}}$ & 0 & 1 & 1 & -1 & 1 \\
\hline Lindley & $\begin{aligned} 1-\frac{(\theta+1+\theta x)}{(\theta+1)} e^{-\theta x} \\
x>0\end{aligned}$ & $(\theta+1+\theta x) e^{-\theta x}$ & 1 & $\frac{-1}{(\theta+1)}$ & 0 & 1 & 1 \\
\hline
\end{tabular}


(Table $\square$ ) contd......

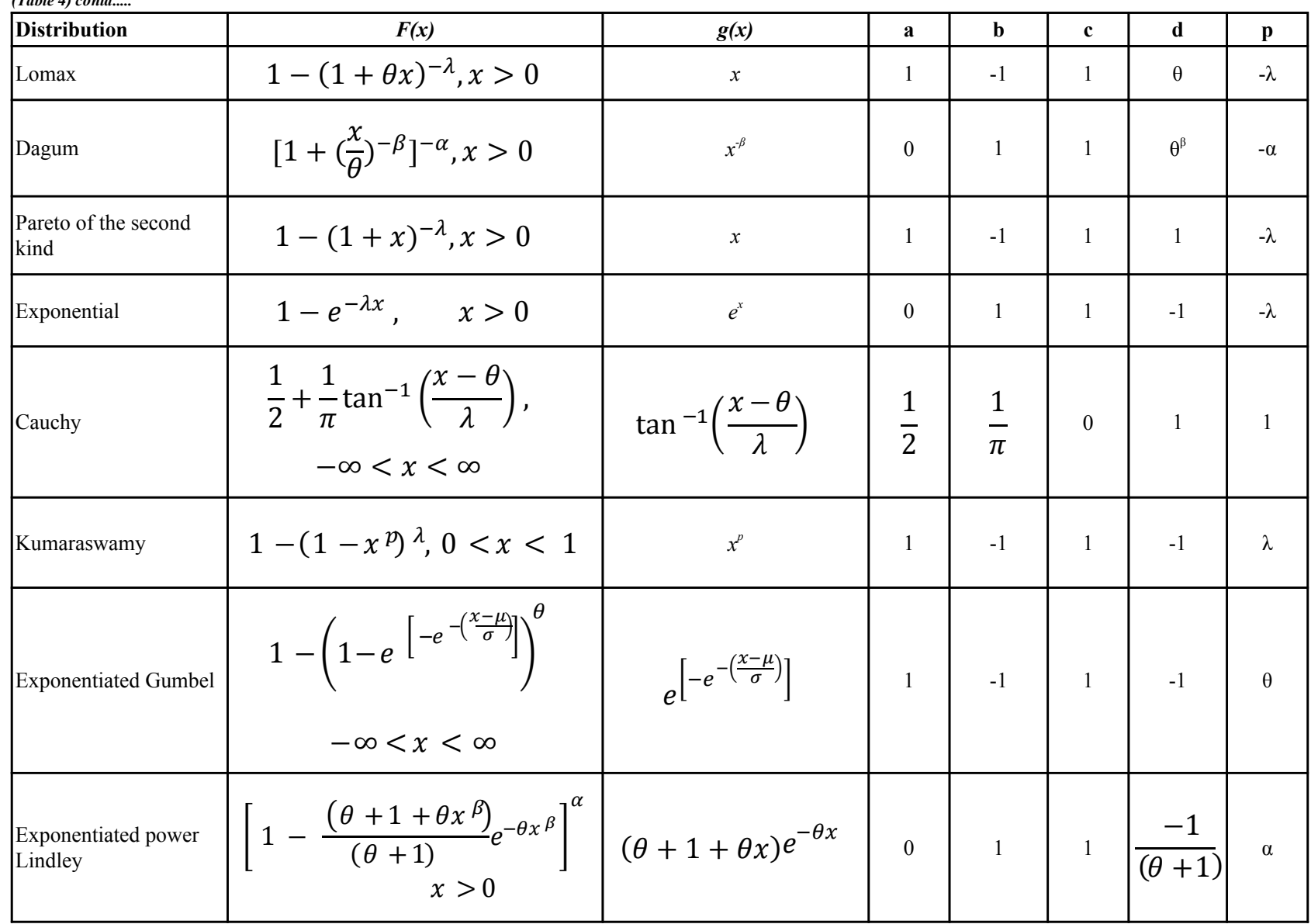

$$
E\left(\left\{g\left(Z_{s}^{(k)}\right)-g\left(Z_{r}^{(k)}\right)\right\}^{p} \mid Z_{r}^{(k)}=x\right)=\xi_{r, s, p}=\frac{\Gamma(p+s-r)}{(k b c)^{p} \Gamma(s-r)} \text { for } \alpha<y<x<\beta
$$

if and only if:

$$
F(x)=\left[a e^{-b g(x)}\right]^{c}
$$

Where $g(x)$ is a continuous, differentiable and non-decreasing function of $\mathrm{x}$ and $\mathrm{p}$ is a positive integer.

\section{Proof:}

For proving the necessary part, from (1) and (2), we have for $\mathrm{s} \geq \mathrm{r}+1$ :

$$
\xi_{r, s, p}=\frac{k^{s-r}}{\Gamma(s-r)} \int_{\alpha}^{\mathrm{X}}\{g(y)-g(x)\}^{p}\left\{\ln \left(\frac{F(x)}{F(y)}\right)\right\}^{s-r-1}\left\{\frac{F(y)}{F(x)}\right\}^{k-1} \frac{f(y)}{F(x)} d y
$$

Using (6), suppose,

$$
\begin{gathered}
w=\ln \left(\frac{F(x)}{F(y)}\right), \quad\{g(y)-g(x)\}^{p}=\left(\frac{w}{b c}\right)^{p} \\
\xi_{r, s, p}=\frac{k^{s-r}}{\Gamma(s-r)} \int_{0}^{\infty}\left(\frac{w}{b c}\right)^{p} w^{s-r-1} e^{-k w} d w=\frac{\Gamma(p+s-r)}{(k b c)^{p} \Gamma(s-r)}
\end{gathered}
$$

Hence the necessary part is proven.

For the sufficiency part, it is clear from (10) that: 


$$
[F(x)]^{k} \xi_{r, s, p}=\frac{k^{s-r}}{\Gamma(s-r)} \int_{\alpha}^{\mathrm{x}}\{g(y)-g(x)\}^{p}\left\{\ln \left(\frac{F(x)}{F(y)}\right)\right\}^{s-r-1}[F(y)]^{k-1} f(y) d y
$$

Differentiating both sides in (11) with respect to $\mathrm{x}$, we get

$$
\begin{aligned}
k f(x)[F(x)]^{k-1} \xi_{r, s, p} & \\
& =\frac{(\mathrm{s}-\mathrm{r}-1) k^{s-r} f(x)}{\Gamma(s-r)} \int_{\alpha}^{\mathrm{x}}\{g(y)-g(x)\}^{p}\left\{\ln \left(\frac{F(x)}{F(y)}\right)\right\}^{s-r-2}[F(y)]^{k-1} \frac{f(y)}{F(x)} d y \\
& -\frac{p k^{s-r} g(x) F(x)}{\Gamma(s-r)} \int_{\alpha}^{\mathrm{x}}\{g(y)-g(x)\}^{p-1}\left\{\ln \left(\frac{F(x)}{F(y)}\right)\right\}^{s-r-1}[F(y)]^{k-1} \frac{f(y)}{F(x)} d y
\end{aligned}
$$

Using (10), we can get that

$$
k f(x) \xi_{r, s, p}=k f(x) \xi_{r+1, s, p}-p \dot{g}(x) F(x) \xi_{r, s, p-1}
$$

and from (9)

$$
p \dot{g}(x) \xi_{r, s, p-1}=k \frac{f(x)}{F(x)}\left(\xi_{r+1, s, p}-\xi_{r, s, p}\right)=\frac{-p f(x)}{b c F(x)} \frac{\Gamma(p+s-r-1)}{(k b c)^{p-1} \Gamma(s-r)}=\frac{-p f(x)}{b c F(x)} \xi_{r, s, p-1}
$$

which gives

$$
\dot{g}(x)=\frac{-f(x)}{b c F(x)}
$$

Therefore, the proof is completed.

\section{Remark:}

When $\mathrm{k}=1$ and $\mathrm{c}=1$, which is the special case of theorem 1 above, has been established in Al-Shomrani and Shawky [17].

\section{Theorem 2:}

Let $\mathrm{X}$ be an absolutely continuous random variables with distribution function $\mathrm{F}(\mathrm{x})$ and $\mathbf{x} \boldsymbol{\epsilon}(\boldsymbol{\alpha}, \boldsymbol{\beta}), F(\alpha)=0$ and $F(\beta)$ $=1$, then

$$
F(x)=\left[a+b e^{-c g(x)}\right]^{d}
$$

if and only if

$$
\mu_{m+1 \mid m}^{(k)}=g(x)+\frac{1}{k c d}+\frac{a}{k b c d} E\left(e^{c g\left(z_{m+1}^{(k)}\right)} \mid Z_{m}^{(k)}=x\right)
$$

Where $b, c$ and $d \neq 0$ are finite constants and $g(x)$ is a continuous, monotonic and differentiable of $\mathrm{x}$ on the support $(\boldsymbol{\alpha}, \boldsymbol{\beta})$.

\section{Proof}

Proving the necessary part, in view of (3), we can get

$$
\mu_{m+1 \mid m}^{(k)}=g(x)-\frac{1}{[F(x)]^{k}} \int_{\alpha}^{x}[F(y)]^{k} d g(y)
$$

Now, from (7), we obtain

$$
f(y)=-b c d \dot{g}^{\prime}(y) e^{-c g(y)}\left[a+b e^{-c g(y)}\right]^{d-1}
$$


which gives

$$
\dot{g}(y)=\frac{-f(y)\left[a+b e^{-c g(y)}\right]}{b c d e^{-c g(y)} F(y)}
$$

Substituting (14) into (13), we get

$$
\mu_{m+1 \mid m}^{(k)}=g(x)+\frac{1}{b c d}\left[a \int_{\alpha}^{x} e^{c g(y)}\left\{\frac{F(y)}{F(x)}\right\}^{k-1} \frac{f(y)}{\overline{F(x)}} d y+b \int_{\alpha}^{x}\left\{\frac{F(y)}{F(x)}\right\}^{k-1} \frac{f(y)}{F(x)} d y\right]
$$

Therefore, (12) is obtained.

For proving the sufficiency part, from (12) and (13), we obtain

$$
\int_{\alpha}^{x}[F(y)]^{k} d g(y)=-\frac{[F(x)]^{k}}{k c d}-\frac{a}{b c d} \int_{\alpha}^{x} e^{c g(y)}[F(y)]^{k-1} f(y) d y
$$

Differentiating both sides in (15) with respect to $\mathrm{x}$, we get

$$
g(x)=\frac{-f(x) e^{c g(x)}\left[a+b e^{-c g(x)}\right]}{b c d F(x)}
$$

Hence, the proof is completed.

\section{Remarks:}

(1) For $\mathrm{k}=1$ and $\mathrm{d}=1$ in theorem 2 above, it has been obtained in Shawky and Abu-Zinadah [18].

(2) For $\mathrm{k}=1$ in theorem 2, it has been addressed in Shawky and Bakoban [19].

(3) If a $=0$ in (11) then $\mu^{(k)}{ }_{m+1 \mid m}=g(x)+\frac{1}{k c d}$.

\section{Theorem 3:}

If $\mathrm{F}(\mathrm{x})<1$ be any cdf of the continuous random variable $\mathrm{X}$ and $\mathrm{x} \boldsymbol{\epsilon}(\boldsymbol{\alpha}, \boldsymbol{\beta}), F(\alpha)=0$ and $F(\beta)=1$, then

$$
F(x)=\left[a+b e^{-c g(x)}\right]^{d}
$$

if and only if

$$
\mu_{m \mid m+1}^{(k)}=g(y)-\frac{H(y)}{b c d(m+1)}\{a \varphi(y)+b\} \text { where } \varphi(y)=E\left(e^{c g\left(z_{m+1}^{(k)}\right)} \mid Z_{m+2}^{(k)}=y\right)
$$

Where $b, c$ and $d \neq 0$ are finite constants and $g(x)$ is a continuous, monotonic and differentiable of $\mathrm{x}$ on the support $(\boldsymbol{\alpha}, \boldsymbol{\beta})$.

\section{Proof:}

For the necessary part, in view of (4), it is straightforward to get

$$
\mu_{m \mid m+1}^{(k)}=g(y)+\frac{1}{[H(y)]^{m}} \int_{y}^{\beta}[H(x)]^{m} \dot{g}(x) d x
$$

Substituting (14) into (17) result in

$$
\mu_{m \mid m+1}^{(k)}=g(y)-\frac{1}{b c d}\left\{a \int_{y}^{\beta} e^{c g(x)}\left[\frac{H(x)}{H(y)}\right]^{m} h(x) d x+b \int_{y}^{\beta}\left[\frac{H(x)}{H(y)}\right]^{m} h(x) d x\right\}
$$


this completes the necessary part.

For the sufficiency part, we have from (16) and (17) that

$$
\int_{y}^{\beta}[H(x)]^{m} \dot{g}(x) d x=\frac{-[H(y)]^{m+1}}{b c d(m+1)}\{a \varphi(y)+b\}
$$

From (16), we get

$$
\dot{\varphi}(y)=\frac{d \varphi}{d y}=\frac{-(m+1) h(y)}{H(y)}\left\{e^{c g(y)}-\varphi(y)\right\}
$$

Differentiating both sides in (18) with respect to y and substituting (19), we have

$$
h(y)=\frac{-b c d \dot{g}(x)}{b+a e^{c g(x)}}
$$

hence, the theorem is proved.

\section{Remarks:}

(1) Fork=1 and $\mathrm{d}=1$ in theorem 3 above, this case has been addressed in Shawky and Abu-Zinadah [18].

(2) Fork $=1$ in theorem 3, it has been obtained in Shawky and Bakoban [19].

\section{Theorem 4:}

Referring to (5) and (8), then

$$
E[g(X) \mid X \leq x]= \begin{cases}g(x)-\frac{a^{2}[c+d g(\alpha)]^{(1-p)}}{b d p F(x)} \operatorname{IBeta}\left(\frac{F(x)}{a} ; 2, \frac{1}{p}\right) & \text { if } a \neq 0 \\ \frac{p g(x)-\frac{c}{d}}{(p+1)} & \text { if } a=0\end{cases}
$$

where $\alpha<x<\beta$ and IBeta $(t ; r, s)=\int_{0}^{t} u^{r-1}(1-u)^{s-1} d u$ is the lower incomplete beta.

\section{Proof:}

If $\mathrm{a} \neq 0$ and from (5) and using integration by parts, we get

$$
E[g(X) \mid X \leq x]=g(x)-\frac{1}{F(x)} \int_{\alpha}^{x} F(w) d g(w)
$$

Now, using (8), we have

this implies

$$
f(w)=b d p \dot{g}(w)[c+d g(w)]^{p-1}
$$

$$
\dot{g}(w)=\frac{f(w)}{b d p[c+d g(w)]^{p-1}}=\frac{f(w)}{b d p\left[\frac{F(w)-a}{b}\right]^{1-\frac{1}{p}}}
$$

Substituting (22) into (21), we get

Let $t=\frac{F(w)}{F(x)}$, then

$$
E[g(X) \mid X \leq x]=g(x)-\frac{\left(\frac{-a}{b}\right)^{\left(\frac{1}{p}-1\right)}}{b d p F(x)} \int_{\alpha}^{x} F(w)\left[1-\frac{F(w)}{a}\right]^{\frac{1}{p}-1} f(w) d w
$$




$$
E[g(X) \mid X \leq x]=g(x)-\frac{[c+d g(\alpha)]^{(1-p)} F(x)}{b d p} \int_{0}^{1} t\left[1-\frac{F(x)}{a} t\right]^{p^{-1}} d t
$$

Where $F(\alpha)=0=a+b[c+d g(\alpha)]^{p} \Rightarrow \frac{-a}{b}=[c+d g(\alpha)]^{p}$

Let $u=\frac{F(x)}{a} t$, then

$$
E[g(X) \mid X \leq x]=g(x)-\frac{a^{2}[c+d g(\alpha)]^{(1-p)}}{b d p F(x)} \int_{0}^{\frac{F(x)}{a}} u[1-u]^{\frac{1}{p}-1} d u
$$

Therefore, the upper part of (20) is achieved.

If $\mathrm{a}=0$ and from (21) and (22) then

$$
E[g(X) \mid X \leq x]=g(x)-\frac{1}{(b)^{\frac{1}{p}} d p F(x)} \int_{\alpha}^{x}[F(w)]^{\frac{1}{p}} f(w) d w
$$

Let $u=\frac{F(w)}{F(x)}$, then

$$
E[g(X) \mid X \leq x]=g(x)-\frac{[F(x)]^{\frac{1}{p}}}{(b)^{\frac{1}{p}} d p} \int_{0}^{1}[u]^{\frac{1}{p}} d u=\frac{p g(x)-\frac{c}{d}}{(p+1)}
$$

Thus, the lower part of (20) is achieved.

\section{Remark:}

The RHS of equation (20) where $\mathrm{a}=0$ is the same as that of equation (2.1.1) in Hamedani, Javanshiri, Maadooliat, and Yazdani [20].

\section{CONCLUSION}

In this study some characterization results and recurrence relations of certain distributions based on the k-th lower record values for three general classes are obtained. Firstly, we characterize the first general form of distributions through conditional expectation of $\mathrm{p}$-th power of difference of functions of two k-th lower record values as shown in Theorem 1. Secondly, two Theorems 2 and 3 are presented for two recurrence relations of the second general class of distributions through conditional expectation of k-th lower record values. Thirdly, we establish an expression of conditional expectation for the third general class of distributions based on truncated moments of some random variable as shown in Theorem 4. Finally, we show examples of some distributions related to these general classes as in Tables $\mathbf{1}$ and 2 .

\section{CONFLICT OF INTEREST}

The author confirms that this article content has no conflict of interest.

\section{ACKNOWLEDGEMENTS}

The author thanks the anonymous referees for their valuable suggestions which let to the improvement of the manuscript.

\section{REFERENCES}

[1] U. Kamps, "Reliability properties of record values from non-identically distributed random variables", Communication in Statistics, Theory and Methods, vol. 23, pp. 2101-2112, 1994.

[http://dx.doi.org/10.1080/03610929408831373]

[2] K.N. Chandler, "The distribution and frequency of record values", Journal of the Royal Statistical Society: Series B, vol. 14, pp. 220-228, 
1952

[3] W. Dziubdziela, and B. Kopociński, "Limiting properties of the k-th record values", Applicationes Mathematicae, vol. 2, pp. 187-190, 1976.

[4] H.N. Nagaraja, "Some characterizations of continuous distributions based on regressions of adjacent order statistics and record values", sankhyaseriesa Sankhya: The Indian Journal of Statistics, Series A (1961-2002), vol. 50, pp. 70-73, 1988.

[5] M. Franco, and J.M. Ruiz, "On characterization of continuous distributions by conditional expectation of record values", sankhyaseriesa Sankhya: The Indian Journal of Statistics, Series A (1961-2002), vol. 58, pp. 135-141, 1996.

[6] M. Franco, and J.M. Ruiz, "On characterizations of distributions by expected values of order statistics and record values with gap", Metrika Metrika: International Journal for Theoretical and Applied Statistics, vol. 45, pp. 107-119, 1997. [http://dx.doi.org/10.1007/BF02717097]

[7] A.H. Khan, and A.A. Alzaid, "Characterization of distributions through linear regression of non-adjacent generalized order statistics", Journal of Applied Statistical Science, vol. 13, pp. 123-136, 2004.

[8] A.H. Khan, M. Faizan, and Z. Haque, "Characterization of continuous distributions through record statistics", Communications of the Korean Mathematical Society, vol. 25, pp. 485-489, 2010. [http://dx.doi.org/10.4134/CKMS.2010.25.3.485]

[9] F.L. Bláquez, and J.L.M. Rebollo, "A characterization of distributions based on linear regression of order statistics and record values", Sankhyā: The Indian Journal of Statistics, Series A, pp. 311-323, 1997.

[10] M. Ahsanullah, Record statistics., Nova Science Publishers: Commack, N.Y., 1995.

[11] M. Ahsanullah, Record values--theory and applications., University Press of America: Dallas, 2004.

[12] B.C. Arnold, N. Balakrishnan, and H.N. Nagaraja, Records., Wiley: New York, 1998. [http://dx.doi.org/10.1002/9781118150412]

[13] V.B. Nevzorov, Records: Mathematical theory., American Mathematical Society: Providence, RI, 2001.

[14] P. Deheuvels, "The characterization of distributions by order statistics and record values a unified approach", Journal of Applied Probability, vol. 21, pp. 326-334, 1984.

[15] H.N. Nagaraja, "Record values and related statistics - a review", Communications in Statistics - Theory and Methods, vol. 17, pp. 2223-2238, 1988. [http://dx.doi.org/10.1080/03610928808829743]

[16] M.Z. Raqab, and A.M. Awad, "Characterizations of the Pareto and related distributions", Metrika Metrika: International Journal for Theoretical and Applied Statistics, vol. 52, pp. 63-67, 2000. [http://dx.doi.org/10.1007/s001840000061]

[17] A.A. Al-Shomrani, and A.I. Shawky, "Some characterization for record values", Acta Scientiae Et Intellectus, vol. 2, pp. 49-56, 2016.

[18] A. Shawky, and H.H. Abu-Zinadah, "General recurrence relations and characterizations of certain distributions based on record values", Journal of Approximation Theory and Applications, vol. 2, pp. 149-159, 2006.

[19] A.I. Shawky, and R.A. Bakoban, "Conditional expectation of certain distributions of record values", International Journal of Mathematical Analysis, vol. 3, pp. 829-838, 2009.

[20] G.G. Hamedani, Z. Javanshiri, M. Maadooliat, and A. Yazdani, "Remarks on characterizations of Malinowska and Szynal", AMC Applied Mathematics and Computation, vol. 246, pp. 377-388, 2014. [http://dx.doi.org/10.1016/j.amc.2014.08.030]

C) Ali A.Al-Shomrani; Licensee Bentham Open

This is an open access article licensed under the terms of the Creative Commons Attribution-Non-Commercial 4.0 International Public License (CC BY-NC 4.0) (https://creativecommons.org/licenses/by-nc/4.0/legalcode), which permits unrestricted, non-commercial use, distribution and reproduction in any medium, provided the work is properly cited. 